\title{
Fetal gut colonization: meconium does not have a detectable microbiota before birth
}

\author{
Katherine M. Kennedy ${ }^{1,4}$, Max J. Gerlach ${ }^{2}$, Thomas Adam³ ${ }^{3}$ Markus M. Heimesaat ${ }^{4}$, Laura Rossi ${ }^{5,6}$, \\ Michael G. Surette ${ }^{1,5,6}$, Deborah M. Sloboda ${ }^{1,6,7 *}$ and Thorsten Braun ${ }^{2 *}$ \\ ${ }^{1}$ Department of Biochemistry and Biomedical Sciences, McMaster University, Hamilton, Canada \\ ${ }^{2}$ Charité - Universitätsmedizin Berlin, corporate member of Freie Universität Berlin, Humboldt- \\ Universität zu Berlin, and Berlin Institute of Health, Department of Obstetrics and Department of \\ 'Experimental Obstetrics', Berlin, Germany \\ ${ }^{3}$ Labor Berlin, Charité Vivantes GmbH, Berlin, Germany \\ ${ }^{4}$ Charité - Universitätsmedizin Berlin, corporate member of Freie Universität Berlin, Humboldt- \\ Universität zu Berlin, and Berlin Institute of Health, Institute for Microbiology, Infectious Diseases and \\ Immunology, Berlin, Germany \\ ${ }^{5}$ Department of Medicine, McMaster University, Hamilton, Canada. \\ ${ }^{6}$ Farncombe Family Digestive Health Research Institute, McMaster University, Hamilton, Canada \\ ${ }^{7}$ Department of Pediatrics, McMaster University, Hamilton, Canada. \\ *shared senior authorship
}

Corresponding Authors

D. M. Sloboda

McMaster University

Department of Biochemistry and Biomedical Sciences

1280 Main St West, Hamilton, Canada

Tel: +1-905-525-9140

Fax: +1-905-522-9033

Email: sloboda@mcmaster.ca

T. Braun

Charité - University Medicine Berlin

Departments of Obstetrics and Division of Experimental Obstetrics

Charité Campus Virchow, Berlin

Germany

Tel: +49-30-664309

Fax: +49-30-564901

Email: thorsten.braun@charite.de 


\section{Abstract}

1 Microbial colonization of the human intestine impacts host metabolism and immunity, however when

2 colonization occurs is unclear. Although numerous studies have reported bacterial DNA in first-pass

3 meconium samples, these samples are collected hours to days after birth. We investigated whether

4 bacteria could be detected in meconium prior to birth. Fetal meconium $(n=20)$ was collected by rectal

5 swab during elective breech Cesarean sections without labour prior to antibiotics and compared to

6 technical and procedural controls $(n=5)$, first-pass meconium (neonatal meconium; $\mathrm{n}=14$ ), and infant

7 stool $(\mathrm{n}=25)$. Unlike first-pass meconium, no microbial signal distinct from negative controls was

8 detected in fetal meconium by $16 \mathrm{~S}$ rRNA gene sequencing. Additionally, positive aerobic $(\mathrm{n}=10$ of 20)

9 and anaerobic $(n=12$ of 20) clinical cultures of fetal meconium (13 of 20 samples positive in at least one

10 culture) were identified as likely skin contaminants, most frequently Staphylococcus epidermidis, and not

11 detected by sequencing in most samples (same genera detected by culture and sequencing in 2 of 13

12 samples with positive culture). We conclude that fetal gut colonization does not occur before birth, and

13 that microbial profiles of neonatal meconium reflect populations acquired during and after birth.

\section{Introduction}

15 Microbial colonization of the human intestine is a key developmental process as the order and timing of

16 microbial exposures shape the development of the gut microbiome ${ }^{1}$ and impact host metabolism and

17 immunity later in life. ${ }^{2}$ In humans, maturation of intestinal barrier function and immunity both occur

18 prenatally. ${ }^{3}$ The fetal intestine is more permeable to macromolecules ${ }^{4}$ and less tolerant of antigens ${ }^{5}$ than

19 that of term infants. Transfer of maternal IgG across the placenta and uptake in the fetal intestine increase

20 near term gestation, ${ }^{6}$ shaping neonatal gut immune responses after birth. ${ }^{7}$ Thus the intrauterine

21 environment has the capacity to shape health well beyond fetal life, and can influence long term health

22 trajectories, ${ }^{8}$ and recently it has been suggested that early-life colonization with specific microbes can 
23 predict health outcomes including asthma ${ }^{9}$ and obesity. ${ }^{10}$ To understand the mechanisms by which

24 microbial colonization influences health later in life we must know when colonization occurs. Several

25 groups using sequencing-based methods have reported bacterial DNA in the placenta ${ }^{11,12}$ and amniotic

26 fluid $^{13}$ and have suggested that this reflects microbial populations that initiate gut colonization in utero. ${ }^{14}$

27 However, recent studies accounting for the high risk of contamination in low-biomass samples ${ }^{15}$ have

28 failed to detect a placental ${ }^{16-18}$ or amniotic fluid microbiome. ${ }^{19-21}$ Thus, this issue remains highly

29 controversial.

As neonatal ("first-pass") meconium is formed prior to birth, it has been used as a proxy for the in utero

31 environment, ${ }^{22,23}$ but this does not account for microbial acquisition that occurs during and/or

32 immediately after birth. Recent metagenomic evidence ${ }^{24}$ and previous culture data ${ }^{25}$ show a correlation

33 between the time from birth to collection and bacterial detection in neonatal meconium. Only one

34 previous study has evaluated the presence of microbes in fetal meconium prior to irth $^{26}$ and found that at mid-gestation the majority of fetal meconium bacterial profiles did not differ from procedural and kidney controls. ${ }^{26}$ Those that did differ were dominated by Micrococcus and Lactobacillus species, likely

37 originating from the maternal cervicovaginal microbiota during sample collection. ${ }^{27}$ Here, we characterized the bacterial profiles of human fetal meconium prior to birth. We show that unlike neonatal meconium, fetal meconium is indistinguishable from negative controls, indicating that colonization of the

40 human gut likely does not occur prior to birth. These data significantly extend our understanding of the

41 establishment of our intestinal microbiome and shed light on which early life influencers may impact

42 postnatal gut health. 


\section{Results}

\section{Participant characteristics}

45 To investigate the possible colonization of the fetal gastrointestinal tract in utero, we analyzed meconium

46 samples collected from 20 term fetuses during caesarean section prior to birth after an intensive method

47 establishment (Table 1). Fetal meconium was sampled by rectal swabbing during elective caesarean

48 section deliveries with no signs of labour, preterm labour, or rupture of membranes to prevent vertical

49 transmission during labour (Fig. 1; Supplementary Fig. 1). As others have previously detected a

50 microbiome in first-pass (neonatal) meconium, ${ }^{2,22}$ we also included neonatal meconium from term

51 deliveries and infant stool samples collected at 6 months of age as positive controls. We included multiple

52 negative controls: a swab exposed to operating room air during caesarean delivery (sampling negative -

53 collected in triplicate for sequencing and cultures during collection of M213), genomic prep reagents

54 either exposed to PCR hood air during sample preparation or not exposed (extraction negatives), and V3-

55 V4 PCR amplifications without added template DNA (PCR negative).

56 Fetal and neonatal participants did not differ significantly in maternal age or birth weight. The gestational

57 age of fetal participants at birth was less than that of neonatal participants ( $p=0.0010$; Welch's t-test),

58 likely due to differences in mode of delivery ( $\mathrm{p}=0.00029, \boldsymbol{\chi}$-squared).

\section{Clinical culture results}

60 Fetal meconium samples and the sampling negative control were cultured under both aerobic and

61 anaerobic conditions (Table 2). Of the 20 fetal meconium samples, 7 were negative for both aerobic and

62 anaerobic cultures after 120 hours, as was the sampling negative control. Additionally, 3 samples had

63 negative aerobic cultures but positive anaerobic cultures (M208, Staphylococcus epidermidis; M210, 
Propionibacterium acnes; M217, Propionibacterium acnes) and 1 sample had a negative anaerobic culture but positive aerobic culture (M219, Staphylococcus epidermidis). Despite growth of Staphylococcus epidermidis under both aerobic and anaerobic conditions only 3 meconium samples had positive cultures under both conditions, while 5 had positive cultures under only one. As

Propionibacterium acnes and coagulase-negative Staphylococci are common skin contaminants in clinical cultures, we attempted to confirm positive results in sequencing data.

\section{S rRNA marker gene sequencing}

We assessed bacterial DNA present in samples using 16S rRNA gene sequencing of combined variable 3 and 4 region (V3V4) amplicons from 30 cycles of PCR amplification. All meconium samples and negative controls were additionally sequenced after 40 PCR cycles to confirm the presence of any genera detected at 30 cycles. We first looked at the number of amplicons sequenced in each sample (read count). Fetal meconium samples were dominated by host-associated reads before removal of host-associated taxa (pruning) (median read count of 109.5; $\min =23 ; \max =1316 ; \mathrm{n}=20$ ), after which they had a median read count of $76.5(\min =16, \max =202, \mathrm{n}=20)$. Read counts of neonatal meconium samples were much more variable: two samples did not have any reads, and the remaining samples had a median read count of 130 $(\min =4, \max =63079, \mathrm{n}=12)$ before pruning and a median read count of 191 after $(\min =9, \max =$ $63079, \mathrm{n}=11)$. Negative controls had a median of 74 reads $(\min =6, \max =396)$ before pruning and a median of 46 reads after $(\min =6, \max =99)$. As expected, infant stool samples had much higher read counts than other samples (median read count of 53207; $\min =5505 ; \max =105212 ; \mathrm{n}=25$ ) before and after pruning.

Looking at within-sample diversity, fetal meconium had lower alpha diversity (Observed ASVs, Shannon index, Simpson's index, Fig. 2A) than infant stool $(p=0.0016, p=0.0001$, and $p=0.0086$ respectively). Neonatal meconium alpha diversity did not differ from that of infant stool $(p=0.7503, p=0.6401$, and $p$ $=0.7762$ respectively). To investigate differences in overall community composition between samples 
(beta-diversity) we performed principal coordinate analysis (PCoA) of Bray-Curtis dissimilarity and found fetal meconium samples cluster with negative controls, while neonatal meconium samples were more variable and more similar to infant stool samples (Fig. 2B). Overall beta-diversity of fetal meconium was indistinguishable from that of negative controls (Fig. 2C) as Bray-Curtis dissimilarity between samples was similar within negative controls, within fetal meconium, and between negative controls and fetal meconium. Compared to fetal meconium, neonatal meconium was more dissimilar to

94 negative controls $(\mathrm{p}<0.001)$.

The most prevalent genera detected in fetal meconium samples were Halomonas (20/20 samples), Rhodanobacter (19/20, only detected in 1 of 2 sequencing runs for 4 samples), and Pseudomonas (15/20, only detected in 1 of 2 sequencing runs for 6 samples), all of which were also detected in negative controls (Fig. 3). The only genera detected in more than one fetal meconium sample and not detected in negative controls were Bacteroides (4/20 samples, only detected in 1 of 2 sequencing runs for 2 samples) and Staphylococcus (4/20 samples, only detected in 1 of 2 sequencing runs for 2 samples). Bacteroides was only consistently detected across sequencing runs and with both 30 and 40 cycles of amplification in

1021 of 20 samples (M202; Supplementary Fig. 2). Staphylococcus was not consistently detected with both

10330 and 40 cycles of PCR amplification in any sample and was only detected by sequencing in 2 of 11

104 samples with positive Staphylococcus culture results (M201 positive for S. epidermidis and M207 for

105 both S. epidermidis and S. lugdunensis). The only other genus consistently detected in a sample with both

10630 and 40 cycles of amplification and across sequencing runs was Escherichia/Shigella (M202, M203,

107 M207) which was also detected in an extraction negative control (Supplementary Fig. 2). Despite culture

108 results positive for Propionibacterium species, no members of this genus were detected in sequencing

109 data from any fetal meconium sample. 


\section{Discussion}

111 The role of the microbiome in controlling host metabolism and its relationship to metabolic dysfunction

112 and obesity ${ }^{28}$ has led investigators to question whether our microbial signatures early in life could be used

113 to predict chronic disease risk. ${ }^{29}$ Because of this, how early in life host-microbe interactions are

114 established has become a topic of intense investigation. In neonates, several microbial species are known

115 to regulate intestinal function ${ }^{30}$ and are key in immune development. ${ }^{31}$ Recent studies reporting

116 colonization may be initiated in utero ${ }^{11,12,14}$ have been the subject of vigorous debate and have been

117 criticized for potential contamination. ${ }^{16,17,21}$ Despite our extensive sample collection optimization to

118 reduce potential contamination (see Methods and Supplementary Fig. 1) more than half of fetal

119 meconium samples had at least one culture test positive for a likely skin contaminant (22/40 total

120 cultures), highlighting the difficulty in avoiding contamination in these types of studies. Contaminating

121 bacteria or bacterial DNA may be introduced by maternal skin or blood during sampling, the environment

122 or investigators during sample handling and culturing, or reagents during DNA extraction and PCR.

123 Negative controls are therefore necessary at each of these stages to rule out contamination. We analyzed

124 fetal meconium collected immediately prior to birth, and compared term fetal meconium to appropriate

125 negative controls, ${ }^{32}$ to neonatal meconium and to infant stool samples. This was a particular strength of

126 our study. Additionally, we used a combination of clinical culture and 16S rRNA gene sequencing to

127 identify a fetal microbial signature. Despite all efforts, we were unable to detect a microbial signature in

128 fetal meconium that was distinct from negative controls. Overall, the lack of a consistent bacterial signal

129 in our data indicates that fetal meconium does not have a microbiome prior to birth.

130 Staphylococci were the dominant genus in culture results (17 cultures) and S. epidermidis was the most

131 prevalent species (11 cultures). In our pilot cohort postoperative swabs of maternal skin around the

132 incision were also positive for S. epidermidis. While it is not possible to know if positive cultures were

133 the result of contamination by skin microbes, the absence of cultured genera in sequencing data make this 
134 a likely possibility. Although S. epidermidis DNA has also been reported in amniotic fluid ${ }^{14}$ and neonatal

135 meconium, ${ }^{24}$ it is most frequently associated with the skin microbiota ${ }^{33}$ suggesting that despite especially

136 stringent efforts to control for contamination some element of contamination may always exist with

137 current technologies and protocols. This makes the strong case for ensuring that a robust set of controls

138 are in place when performing these types of investigations.

139 Although sequencing cannot prove the absence of bacterial DNA in a sample, we used robust negative

140 controls and technical replicates to distinguish contamination signals from stochastic sequencing noise.

141 While previous studies have reported the existence of bacterial DNA in amniotic fluid and the placenta,

142 these studies lacked sequencing data from negative controls and therefore cannot rule out

143 contamination. ${ }^{15}$ In our study, we included negative controls collected during sampling, extraction, and

144 PCR amplification. Inclusion of these negative controls allowed us to identify the most prevalent and

145 abundant genera as likely contaminants introduced during extraction (Halomonas and Rhodanobacter) or

146 sampling (Pseudomonas). Additionally, we included neonatal meconium as a positive control of our

147 extraction and amplification of bacterial DNA from these low-biomass samples.

148 Even when sequencing data from appropriate negative controls are included, low-biomass samples are 149 especially sensitive to stochastic amplification ${ }^{34}$ and sequencing ${ }^{32}$ noise. Determination of a true bacterial

150 signal requires its presence across technical replicates. In this study, we ran the PCR products of each

151 amplification on two separate sequencing runs. Excluding likely contaminants, almost all genera detected

152 were only found in one of two sequencing runs for each sample. Bacteroides was the only genus

153 consistently detected across technical replicates, and this was only true in 1 of 20 fetal meconium

154 samples. Thus, when comparing across sequencing runs, we found that the bacterial signals in fetal

155 meconium that are not also detected in negative controls are likely due to stochastic sequencing noise.

156 In terms of overall community composition, fetal meconium beta-diversity was similar to that of negative

157 controls and lower than that of neonatal meconium. Neonatal meconium community composition was 
158 highly variable with some samples more closely resembling infant stool. Thus, microbial profiles of

159 neonatal meconium reflect populations acquired during and after birth and not populations that exist prior

160 to birth.

161 To our knowledge, this is the first study to investigate the meconium microbiome in term human neonates

162 prior to birth and this is also the first study to control for both contamination during sampling and DNA

163 extraction and stochastic noise during sequencing. While it is not possible to prove the absence of bacteria

164 in fetal meconium prior to birth, our data do not support in utero colonization. These data suggest that

165 colonization more likely occurs either during birth via maternal skin/vaginal/fecal seeding, or post birth

166 via environmental seeding.

167 Methods

168 Study design and sample collection

170 A pilot cohort of 22 participants was recruited to optimize sample collection methods to minimize risk of

171 contamination and comprised two pilot cohorts as described below (Pilot 1a and 1b). Only caesarean

172 deliveries were included to avoid the vertical transmission of bacteria during a vaginal birth, during which

173 the child is exposed to the maternal bacterial flora. In addition to the duration of the birth process itself, a

174 further disadvantage of spontaneous delivery is the variable time within which the newborn engages its

175 first bowel movement (neonatal meconium - which is of prenatal origin). Study inclusion was further

176 restricted to elective caesarean sections prior to labour due to potential microbial influences on unplanned

177 section deliveries and to guarantee the presence of an operator familiar with the study protocol and an

178 equally trained research personnel who was able to carry out the immediate transport and further 
179 processing of the sample in the nearby laboratory. Breech presentations were preferred to ensure that the

180 rectum was immediately accessible for sampling after sectioning and before delivery and to minimize

181 manipulation of the child before sampling (Fig. 1). This ensured a reduced risk of contamination as a

182 swab could be taken before the neonate was fully removed from the uterus. Finally, to avoid false-

183 negative results the preoperative prophylactic antibiotic was administered after meconium sample

184 collection.

185 Pilot cohort 1a (establishment of sampling procedures)

186 Swabs with nylon flock fiber (eSwab TM, Copan Diagnostics Inc.) were used to collect fetal meconium

187 samples. In the event of a meconium leakage (spontaneous as a side effect of breech presentation), a

188 sample was either taken up with a swab, or a careful rectal smear was performed. Pilot samples were

189 transferred to blood culture bottles (BD BACTEC ${ }^{\mathrm{TM}}$ ) and brought to the Charité University Berlin

190 laboratory for culturing. The inoculation of the blood culture bottles was initially not carried out under

191 sterile conditions, but the use of a sterile workbench was quickly considered and tested (from \#4 onward,

192 not continuously). An amniotic fluid sample (\#9) and fetal perianal swabs (\#6, \#7) were also cultured to

193 identify possible sources of contamination. The Head of Clinical Microbiology from Labor Berlin was

194 consulted for an external review of the study design and protocol. Of the 16 pilot meconium cultures (8

195 aerobic +8 anaerobic), 11 showed bacterial detection (68.8\%), 2 of which were more than one species

196 (\#2,\#10). Only 1 of the 8 pilot samples (\#5) had both anaerobic and aerobic negative cultures (12.5\%), 3

197 cases $(\# 2, \# 6, \# 8)$ had only anaerobic positive cultures $(37.5 \%)$ and 4 cases $(\# 3 . \# 4, \# 7, \# 10)$ had both

198 anaerobic and aerobic positive cultures (50\%). Positive cultures had a median incubation time of 20 hours

199 until detection. The 13 detected microbial populations could be assigned to the following species in

200 decreasing frequency: Staphylococcus epidermidis (5), Staphylococcus lugdunensis (3), Staphylococcus

201 capitis (2), Staphylococcus caprae, Propionibacterium acnes, Propionibacterium avidum (1 each). The

202 amniotic fluid sample showed no contamination. Staphylococcus epidermidis (both anaerobic and 
aerobic, \#7), Enterobacter aerogenes (anaerobic, \#6), and Staphylococcus lugdunensis (aerobic, \#6) were

204 detected in perianal swabs. Maternal skin was considered as the potential source of contamination, and

205 therefore a second pilot study was performed to minimize skin contamination during sampling.

206 Pilot cohort $1 \mathrm{~b}(\# 11-\# 22)$

207 To investigate maternal skin as a potential source of contamination, we cultured a set of pilot swabs of

208 maternal skin around the caesarean section area both preoperatively after disinfection and postoperatively

209 after the end of wound care (\#11). These samples were collected and cultured as above in pilot 1a.

210 Anaerobic preoperative swab cultures were positive for Staphylococcus lugdenensis, Bacillus

211 licheniformis, and Clostridium perfringens. Postoperative swab cultures were positive for Staphylococcus

212 epidermidis (both anaerobic and aerobic) and Bacillus licheniformis (anaerobic) and Staphylococcus

213 lugdunensis (aerobic). To reduce contamination from maternal skin microbes, we expanded the area of

214 disinfection with Softasept ${ }^{\mathrm{R}} \mathrm{N}$ coloured from the maternal armpits to her knees. To reduce the chance of

215 contamination due to amniotic fluid dampening the surgical draping, a new model of draping was

216 designed and used (from \#15 on) that had a wider design, better adhesive properties, and a thinner film.

217 The thinner film helped in cases where the mother had repeated caesarean sections, where the prior model

218 sometimes required the film to be slit to visualize the existing scar. The film of the new perforated sheet

219 remained intact and the aseptic area remained undisturbed up to the skin incision. Lateral drapes were

220 also introduced (from \#16 on) and the remaining pilot samples were used to observe the resistance of the

221 seal, to identify possible points susceptible to film detachments (\#17 - \#22).

\section{Principal Study cohort}

223 Participants ( $\mathrm{n}=19$ mothers) were recruited during routine antenatal counselling when patients presented

224 with planned caesarean section and breech presentation at $36+0$ weeks gestation. The study protocol was 
225 reviewed and approved by Charité ethics committee (EA 04/059/16). Informed consent was obtained

226 from all study participants.

227 Advanced physical disinfection of maternal skin was performed (as described in pilot 1b), covering the

228 area from maternal armpits to knees and the surgical area was covered using the specialized sterile drape,

229 with film remaining intact until the incision. After caesarean section and exposure of the fetal buttocks

230 prior to birth, meconium was rectally sampled using sterile eSwabs ${ }^{\mathrm{TM}}$. At least one operating room

231 negative control (swab exposed to operating room air) was also taken. Triplicate samples were collected,

232 of which two were immediately cultured (anaerobic and aerobic) and one was flash frozen in liquid

233 nitrogen for future sequencing.

\section{Neonatal meconium and infant stool sample collection}

235 Neonatal meconium and infant stool samples used as positive controls were collected as part of a separate

236 study (unpublished). Neonatal meconium samples were collected from diapers within 48 hours of birth

237 and frozen at $-80^{\circ} \mathrm{C}$ prior to processing for sequencing. Infant stool samples were collected at 6 months of

238 age by parents and frozen at $-20^{\circ} \mathrm{C}$ before being stored at $-80^{\circ} \mathrm{C}$ prior to processing for sequencing.

239 Neonatal meconium and infant stool samples were not included in bacterial culturing.

\section{Bacterial culturing}

241 Samples were transferred in a sterile hood to anaerobic and aerobic point bottles (BD Bactec system ${ }^{\mathrm{TM}}$ )

242 and supplemented (BD BACTEC TM FOS TM) to improve the growth conditions for more demanding

243 bacterial species. Cultures were maintained for a maximum of 120 hours. Positive cultures were sub-

244 cultured on agar plates and identified using MALDI-TOF (VitekMS, bioMérieux). 


\section{DNA extraction and amplification}

246 Sample aliquots were transferred to genomic prep tubes in a sterile PCR hood using sterile biopsy

247 punches and gloves were changed between handling each sample. In addition to the sampling negative

248 control collected in the operating room, an extraction negative control (opened prep tube in the PCR hood

249 during sample aliquoting) was included. An additional extraction negative control prep tube remained

250 closed during sample aliquoting. Genomic DNA was extracted as previously described ${ }^{35}$ with the addition

251 of a mechanical lysis step using $0.2 \mathrm{~g}$ of $2.8 \mathrm{~mm}$ ceramic beads to improve extraction efficiency and

252 without mutanolysin. PCR amplification of the variable 3 and 4 (V3-V4) regions of the 16S rRNA gene

253 was subsequently performed on the extracted DNA from each sample using methods previously

254 described. ${ }^{36}$ In addition to the typical 30 cycles of amplification, each sample was also independently

255 amplified for 40 cycles to confirm any signals detected - these additional amplifications were not used for

256 general analysis as amplification beyond 30 cycles is known to bias sequencing data. ${ }^{37}$ Each reaction

257 contained 5 pmol of primer (341F - CCTACGGGNGGCWGCAG, 806R - GGACTACNVGGGTWTC-

258 TAAT), $200 \mathrm{mM}$ of dNTPs, $1.5 \mu 150 \mathrm{mM} \mathrm{MgCl}_{2}, 2 \mu \mathrm{l}$ of $10 \mathrm{mg} / \mathrm{ml}$ bovine serum albumin (irradiated

259 with a transilluminator to eliminate contaminating DNA) and $0.25 \mu 1$ Taq polymerase (Life Technologies,

260 Canada) for a total reaction volume of $50 \mu 1.341 \mathrm{~F}$ and $806 \mathrm{R}$ rRNA gene primers were modified to

261 include adapter sequences specific to the Illumina technology and 6-base pair barcodes were used to

262 allow multiplexing of samples. 16S DNA products of the PCR amplification were subsequently

263 sequenced twice on two separate runs of the Illumina MiSeq platform (2x300bp) at the Farncombe

264 Genomics Facility (McMaster University, Hamilton ON, Canada).

\section{16S rRNA gene sequencing and analysis}

266 Primers were trimmed from FASTQ files using Cutadapt ${ }^{38}$ (RRID:SCR_011841) and DADA2 ${ }^{39}$ was used

267 to derive amplicon sequence variants (ASVs). Taxonomy was assigned using the Silva 132 reference 
268 database. $^{40}$ Non-bacterial ASVs were culled (kingdom Eukaryota, family Mitochondria, order

269 Chloroplast, or no assigned phylum), as was any ASV to which only 1 sequence was assigned.

270 We performed alpha and beta diversity analyses in R using phyloseq ${ }^{41}$ (RRID:SCR_013080) and tested

271 for whole community differences across groups using vegan's ${ }^{42}$ (RRID:SCR_011950) implementation of

272 permutational multivariate analysis of variance (PERMANOVA) in the adonis command. These results

273 were visualized via Principal Coordinate Analysis (PCoA) ordination using R's ggplot2 package

274 (RRID:SCR_014601). ${ }^{43}$ ASV sequences were aligned using DECIPHER ${ }^{44}$ and a GTR + G + I (Generalized

275 time-reversible with Gamma rate variation) maximum likelihood tree phylogenetic tree was constructed

276 with phangorn ${ }^{45}$ using a neighbor-joining tree as a starting point. Significance of alpha diversity was

277 analyzed by linear mixed model with sample type as a fixed effect and participant ID as a random effect.

278 Significance of Bray-Curtis distances between and within sample types was assessed by linear mixed

279 model with sample type comparison as a fixed effect (i.e. neg-neg, neg-fetal, etc.) and participant ID as a

280 random effect.

281 Data availability

282 All sequencing data associated with this study has been made publicly available. 16S rRNA bacterial 283 profiling data generated in this study is available in the NCBI Sequence Read Archive under project ID

284 PRJNA666699. 


\section{References}

286 1. Sprockett, D., Fukami, T. \& Relman, D. A. Role of priority effects in the early-life assembly of the gut microbiota. Nat. Rev. Gastroenterol. Hepatol. 15, 197-205 (2018).

2. Durack, J. et al. Delayed gut microbiota development in high-risk for asthma infants is temporarily modifiable by Lactobacillus supplementation. Nat. Commun. 9, 707 (2018).

3. Weström, B., Arévalo Sureda, E., Pierzynowska, K., Pierzynowski, S. G. \& Pérez-Cano, F.-J. The immature gut barrier and its importance in establishing immunity in newborn mammals. Front. Immunol. 11, 1153 (2020).

4. Axelsson, I. et al. Macromolecular absorption in preterm and term infants. Acta Paediatr. Scand. 78, 532-537 (1989).

5. Nanthakumar, N. et al. The mechanism of excessive intestinal inflammation in necrotizing enterocolitis: an immature innate immune response. PLoS One 6, e17776 (2011).

6. Chen, K., Magri, G., Grasset, E. K. \& Cerutti, A. Rethinking mucosal antibody responses: IgM, IgG and IgD join IgA. Nat. Rev. Immunol. 20, 427-441 (2020).

7. Yoshida, M. et al. Human neonatal Fc receptor mediates transport of IgG into luminal secretions for delivery of antigens to mucosal dendritic cells. Immunity 20, 769-783 (2004).

8. Hanson, M. A. \& Gluckman, P. D. Developmental origins of health and disease: new insights. Basic Clin. Pharmacol. Toxicol. 102, 90-93 (2008).

303 9. Fujimura, K. E. et al. Neonatal gut microbiota associates with childhood multisensitized atopy and T cell differentiation. Nat. Med. 22, 1187-1191 (2016).

305 10. Soderborg, T. K. et al. The gut microbiota in infants of obese mothers increases inflammation and susceptibility to NAFLD. Nat. Commun. 9, 4462 (2018).

307 11. Aagaard, K. et al. The placenta harbors a unique microbiome. Sci. Transl. Med. 6, 237ra65 (2014).

308 12. Antony, K. M. et al. The preterm placental microbiome varies in association with excess maternal 309 gestational weight gain. Am. J. Obstet. Gynecol. 212, 653.e1-16 (2015). 
13. Stinson, L. et al. Comparison of Bacterial DNA Profiles in Mid-Trimester Amniotic Fluid Samples From Preterm and Term Deliveries. Front. Microbiol. 11, 415 (2020).

14. Collado, M. C., Rautava, S., Aakko, J., Isolauri, E. \& Salminen, S. Human gut colonisation may be initiated in utero by distinct microbial communities in the placenta and amniotic fluid. Sci. Rep. $\mathbf{6}$, 23129 (2016).

15. Salter, S. J. et al. Reagent and laboratory contamination can critically impact sequence-based microbiome analyses. BMC Biol. 12, 87 (2014).

16. Lauder, A. P. et al. Comparison of placenta samples with contamination controls does not provide evidence for a distinct placenta microbiota. Microbiome 4, 29 (2016).

319 17. Theis, K. R. et al. Does the human placenta delivered at term have a microbiota? Results of 320 cultivation, quantitative real-time PCR, 16S rRNA gene sequencing, and metagenomics. Am. J. $321 \quad$ Obstet. Gynecol. 220, 267.e1-267.e39 (2019).

322 18. Olomu, I. N. et al. Elimination of "kitome" and "splashome" contamination results in lack of detection of a unique placental microbiome. BMC Microbiol. 20, 157 (2020).

324 19. Lim, E. S., Rodriguez, C. \& Holtz, L. R. Amniotic fluid from healthy term pregnancies does not 325 harbor a detectable microbial community. Microbiome 6, 87 (2018).

326 20. Rehbinder, E. M. et al. Is amniotic fluid of women with uncomplicated term pregnancies free of 327 bacteria? Am. J. Obstet. Gynecol. 219, 289.e1-289.e12 (2018).

328 21. de Goffau, M. C. et al. Human placenta has no microbiome but can contain potential pathogens. $329 \quad$ Nature 572, 329-334 (2019).

330 22. Wang, J. et al. Dysbiosis of maternal and neonatal microbiota associated with gestational diabetes $331 \quad$ mellitus. Gut (2018) doi:10.1136/gutjnl-2018-315988.

332 23. Hu, J. et al. Diversified microbiota of meconium is affected by maternal diabetes status. PLoS One 8, 333 e78257 (2013).

334 24. Bittinger, K. et al. Bacterial colonization reprograms the neonatal gut metabolome. Nat. Microbiol. $335 \quad 5,838-847$ (2020). 
25. Hall, I. C. Bacterial flora of first specimens of meconium passed by fifty new-born infants. Arch. Pediatr. Adolesc. Med. 47, 1279 (1934).

26. Rackaityte, E. et al. Viable bacterial colonization is highly limited in the human intestine in utero. Nat. Med. (2020) doi:10.1038/s41591-020-0761-3.

27. Chen, C. et al. The microbiota continuum along the female reproductive tract and its relation to uterine-related diseases. Nat. Commun. 8, 875 (2017).

28. Moreno-Indias, I., Cardona, F., Tinahones, F. J. \& Queipo-Ortuño, M. I. Impact of the gut microbiota on the development of obesity and type 2 diabetes mellitus. Front. Microbiol. 5, 190 (2014).

29. Gohir, W., Ratcliffe, E. M. \& Sloboda, D. M. Of the bugs that shape us: maternal obesity, the gut microbiome, and long-term disease risk. Pediatr. Res. 77, 196-204 (2015).

30. Singer, J. R. et al. Preventing dysbiosis of the neonatal mouse intestinal microbiome protects against late-onset sepsis. Nat. Med. 25, 1772-1782 (2019).

31. Galazzo, G. et al. Development of the Microbiota and Associations With Birth Mode, Diet, and Atopic Disorders in a Longitudinal Analysis of Stool Samples, Collected From Infancy Through Early Childhood. Gastroenterology (2020) doi:10.1053/j.gastro.2020.01.024.

32. Erb-Downward, J. R. et al. Critical Relevance of Stochastic Effects on Low-Bacterial-Biomass 16S rRNA Gene Analysis. MBio 11, (2020).

33. Stacy, A. \& Belkaid, Y. Microbial guardians of skin health. Science 363, 227-228 (2019).

34. Kennedy, K., Hall, M. W., Lynch, M. D. J., Moreno-Hagelsieb, G. \& Neufeld, J. D. Evaluating bias of illumina-based bacterial 16S rRNA gene profiles. Appl. Environ. Microbiol. 80, 5717-5722 (2014).

35. Whelan, F. J. et al. The loss of topography in the microbial communities of the upper respiratory tract in the elderly. Ann. Am. Thorac. Soc. 11, 513-521 (2014).

36. Bartram, A. K., Lynch, M. D. J., Stearns, J. C., Moreno-Hagelsieb, G. \& Neufeld, J. D. Generation of multimillion-sequence $16 \mathrm{~S}$ rRNA gene libraries from complex microbial communities by 
assembling paired-end illumina reads. Appl. Environ. Microbiol. 77, 3846-3852 (2011).

363
37. Sze, M. A. \& Schloss, P. D. The impact of DNA polymerase and number of rounds of amplification in PCR on 16S rRNA gene sequence data. mSphere 4, (2019).

38. Martin, M. Cutadapt removes adapter sequences from high-throughput sequencing reads. EMBnet.journal 17, 10-12 (2011).

39. Callahan, B. J. et al. DADA2: High-resolution sample inference from Illumina amplicon data. Nat. Methods 13, 581-583 (2016).

40. Quast, C. et al. The SILVA ribosomal RNA gene database project: improved data processing and web-based tools. Nucleic Acids Res. 41, D590-6 (2013).

41. McMurdie, P. J. \& Holmes, S. phyloseq: an R package for reproducible interactive analysis and graphics of microbiome census data. PLoS One 8, e61217 (2013).

42. Oksanen, J. et al. Vegan: Community Ecology Package. 2013. R-package version 2.0-10. (2013).

43. Wickham, H. ggplot2: Elegant Graphics for Data Analysis. (Springer, 2016).

44. Wright, E. S. RNAconTest: comparing tools for noncoding RNA multiple sequence alignment based on structural consistency. RNA 26, 531-540 (2020).

45. Schliep, K. P. phangorn: phylogenetic analysis in R. Bioinformatics 27, 592-593 (2011). 


\section{Acknowledgements}

379 We thank all the participants that were recruited in this study. We would like to thank Dr. Hanna

380 Brinkmann, Laura Pasura, Laura Maschirow and Alexander Schwickert for assisting with patient

381 recruitments; Mrs. Loreen Ehrlich with sample preparation and Dr. Katharina von Weizsaecker for her

382 external review of the microbiology protocol and advice on protocol improvements. We thank Michelle

383 Shah for performing genomic DNA extractions. K.M.K. is supported by a Farncombe Digestive Health

384 Research Institute Student Fellowship. MGS and DMS are supported by the Canada Research Chairs

385 Program.

386 Author Contributions

387 K.M.K. analyzed sequencing data and wrote the manuscript. M.G. contributed to sample collection and

388 wrote the Study Design and Sample Collection portion of the Methods section. T.A. performed the

389 culture-based analyses. M.M.H. assisted in study design. L.R. assisted in study design and performed

390 V3V4 amplifications and processing of raw sequencing data. M.G.S. assisted in study design and analysis

391 of sequencing data. D.M.S. contributed to data analysis, and manuscript development. T.B. designed the

392 study and contributed to sample collection. All authors discussed the analyses and results and edited the

393 manuscript.

394 Competing Interests statement

395 The authors declare that they have no competing interests. 


\section{Figure Legends}

Figure 1. Diagram of collection method for fetal meconium samples. Following advanced physical disinfection of maternal skin from maternal armpits to knees, the surgical area was covered using the specialized sterile drape, with film remaining intact until the incision. After caesarean section and exposure of the fetal buttocks prior to birth, meconium was rectally sampled using sterile eSwabs ${ }^{\mathrm{TM}}$. meconium (fetal, $\mathrm{n}=20$ ), neonatal meconium ( assessed by a linear mixed model, with sample type as a fixed effect and participant ID as a random effect $(\mathrm{p}<0.05$ indicated by $*)$. $b$, Principal coordinate analysis $(\mathrm{PCoA})$ of Bray-Curtis distances at genus level shows fetal meconium samples (blue dots) cluster with sampling negative controls (red), indicating their community composition is similar, while neonatal meconium samples (purple) are more variable. c, Boxplots of Bray-Curtis dissimilarities within (red) and between (black) sample types shows that fetal meconium samples less dissimilar to each other and similar to negative controls. Significance assessed by

410 linear mixed model with sample type comparison as a fixed effect (i.e. neg-neg, neg-fetal, etc.) and

411 participant ID as a random effect. Boxplot center line, median; box limits, upper and lower quartiles;

412 whiskers, $1.5 \mathrm{x}$ interquartile range; points, outliers.

413 Figure 3. Neighbour-joining phylogenetic tree of all genera and presence/absence in each sample.

414 Taxonomy was assigned using the RDP Classifier against the Silva 132 reference database. Dots indicate

415 presence of the genera at any abundance in the given sample. Gammaproteobacteria prevalent in fetal

416 meconium (fetal) are also prevalent in sampling and extraction negative controls (neg). Microbial profiles

417 of first-pass neonatal meconium (neo) share more genera with those of infant stool (inf) than fetal

418 meconium.

\section{Supplementary Figure 1. Optimization of collection to reduce contamination.}

420 Supplementary Figure 2. Detection of genera across technical replicates. The abundance (read count)

421 is shown for each sample by sequencing run (run 1 and run 2) for 30 cycles of PCR amplification. Genera

422 are only shown if they were also detected within the same sample's sequencing data from 40 cycles of

423 PCR amplification. 


\section{Tables}

\begin{tabular}{lll}
\hline \multicolumn{3}{l}{ Table 1. Participant characteristics } \\
\hline & $\begin{array}{l}\text { Fetal } \\
(\mathrm{n}=20)\end{array}$ & $\begin{array}{l}\text { Neo } \\
(\mathrm{n}=14)\end{array}$ \\
\hline $\begin{array}{l}\text { Maternal age, years } \\
\text { mean (SD) }\end{array}$ & $32.3(5.1)$ & $33.2(4.4)$ \\
\hline $\begin{array}{l}\text { Gestational age, days * } \\
\text { mean (SD) }\end{array}$ & $271.4(5.6)$ & $276.7(6.0)$ \\
\hline $\begin{array}{l}\text { Birth weight, grams } \\
\text { mean (SD) }\end{array}$ & $3240(380)$ & $3384(557)$ \\
\hline Sex & $14(70)$ & $7(47)$ \\
\hline Female & $6(30)$ & $8(53)$ \\
\hline Male & $0(0)$ & $9(60)$ \\
\hline Delivery, $\mathrm{n}(\%)^{+}$ & $8(40)$ \\
\hline Vaginal & $20(100)$ & \\
\hline Caesarean & & \\
\hline $\mathrm{p}<0.05$ indicated by * (t-test) or ${ }^{+}$Chi-squared \\
\hline
\end{tabular}

\begin{tabular}{|c|c|c|c|c|}
\hline \multirow[b]{2}{*}{ ID } & \multicolumn{2}{|c|}{ Anaerobic culture } & \multicolumn{2}{|l|}{ Aerobic culture } \\
\hline & Result & $\begin{array}{l}\text { Incubation } \\
\text { Time (hrs) }\end{array}$ & Result & $\begin{array}{l}\text { Incubation } \\
\text { Time (hrs) }\end{array}$ \\
\hline M201 & Staph. epidermidis & 18.64 & Staph. epidermidis & 23.2 \\
\hline M202 & Staph. epidermidis & 18.68 & Staph. lugdunensis & 20.67 \\
\hline M203* & Staph. epidermidis & 20.12 & Staph. epidermidis & 14.92 \\
\hline M204*† & Staph. epidermidis & 18.59 & Staph. epidermidis & 17.09 \\
\hline M205† & negative & 120.07 & negative & 120.07 \\
\hline M206 & Staph. saprophyticus & 26.09 & Staph. saprophyticus & 17.96 \\
\hline $\mathbf{M 2 0 7 \dagger}$ & Staph. epidermidis & 19.06 & Staph. lugdunensis & 18.69 \\
\hline M208 & Staph. epidermidis & 22.05 & negative & 120.08 \\
\hline M209 & negative & 120.07 & negative & 120.08 \\
\hline M210 & Propionibacterium acnes & 79.21 & negative & 120.08 \\
\hline M211 & negative & 120.08 & negative & 120.09 \\
\hline M212 & Staph. epidermidis & 19.94 & Staph. hominis & 24.29 \\
\hline M213 & Propionibacterium acnes & 102.77 & Staph. capitis & 36.68 \\
\hline Neg & negative & 102.02 & negative & 120.03 \\
\hline M215 & negative & 120.13 & negative & 120.13 \\
\hline M216 & negative & 120.16 & negative & 120.15 \\
\hline M217 & Propionibacterium acnes & 66.24 & negative & 120.17 \\
\hline M219 & negative & 120.08 & Staph. epidermidis & 17.41 \\
\hline M221 & negative & 120.08 & negative & 120.08 \\
\hline M222 & negative & 120.08 & negative & 120.09 \\
\hline M223 & Propionibacterium avidum & 80.85 & Propionibacterium avidum & 99.05 \\
\hline
\end{tabular}


-Expanded area of maternal disinfection

-Specialized surgical drape intact until incision

-Breech caesarean sections minimize manipulation 

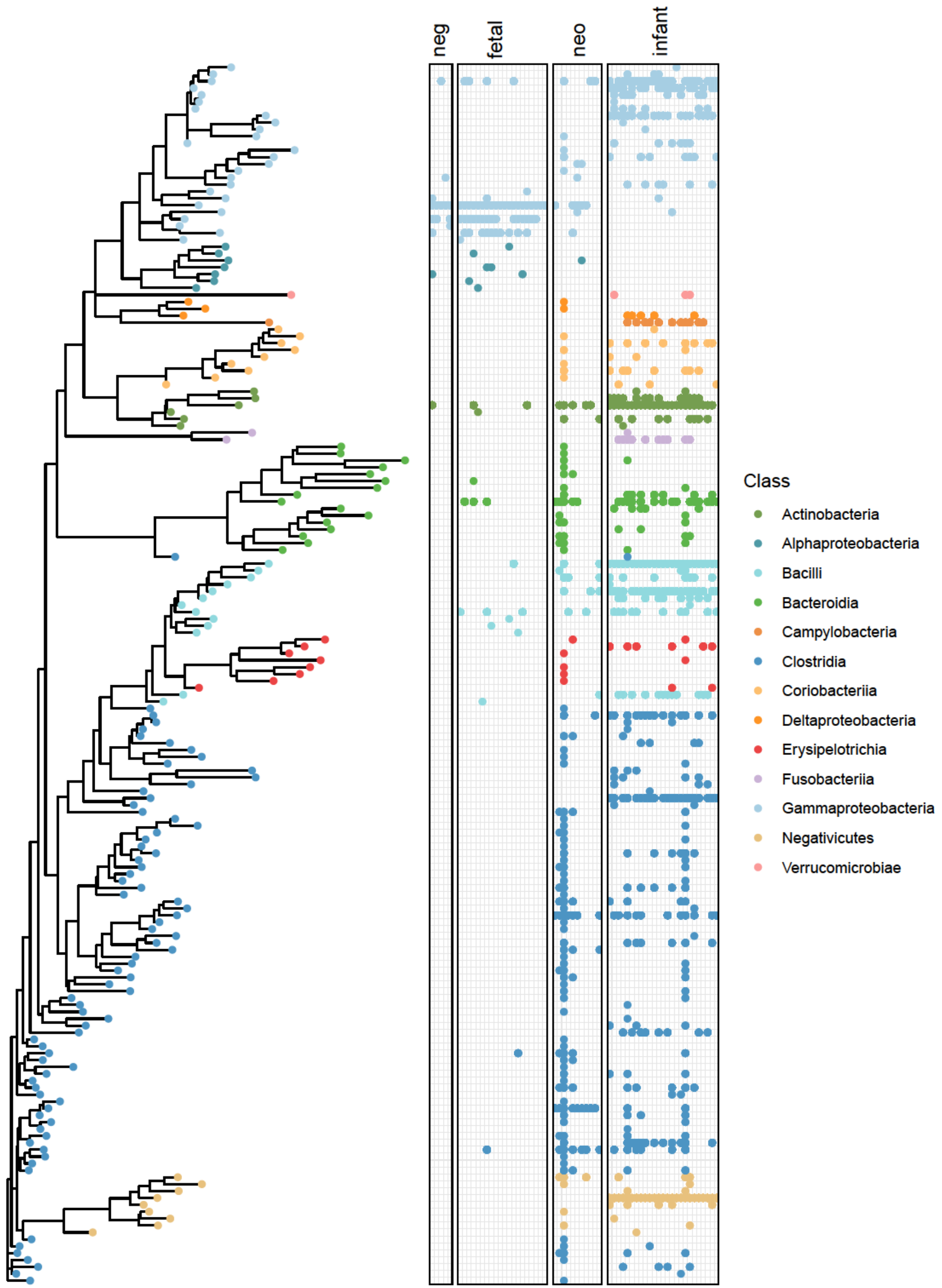


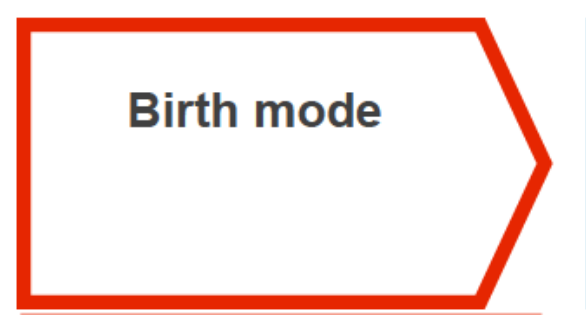

- Problem: vertical

contamination during

vaginal delivery $\Rightarrow$

Solution: caesarean

section

- Problem: Infectious etiology in secondary caesarean section $\Rightarrow$ Solution: primary caesarean section

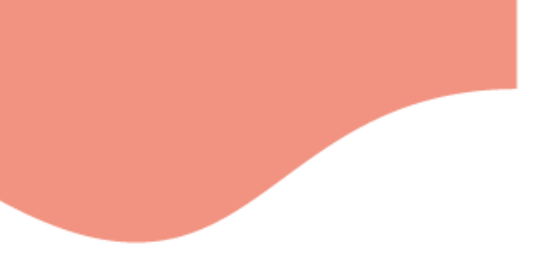

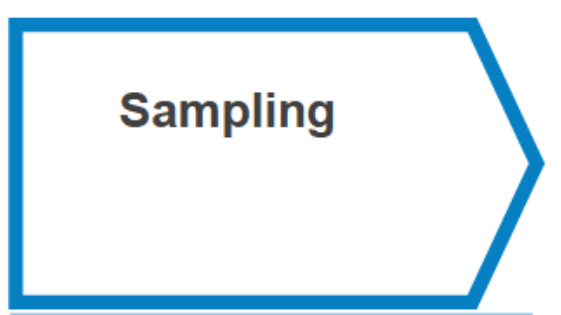

\section{- Problem:}

Contamination from manipulation of the child after development $\Rightarrow$ Solution: breech deliveries

- Problem: contamination of the surgical field $\Rightarrow$ Solution: extended aseptic area, special ad sheet

- Problem: false-negative results through preoperative antibiotic treatment $\Rightarrow$ Solution: antibiotic prophylaxis after sampling

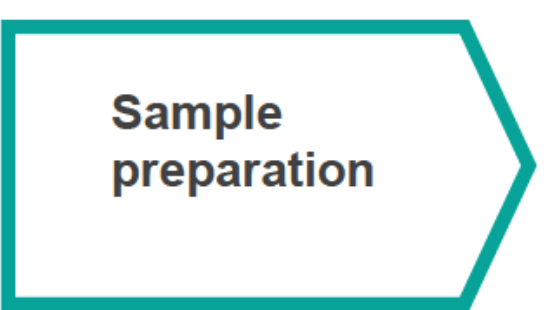

- Problem: incubation time of possible contaminants $\Rightarrow$ Solution: Timely processing (scheduling laboratory on campus)

- Problem: contamination through laboratory processing $\Rightarrow$ Solution: Disinfection; sterile workbench; Material change

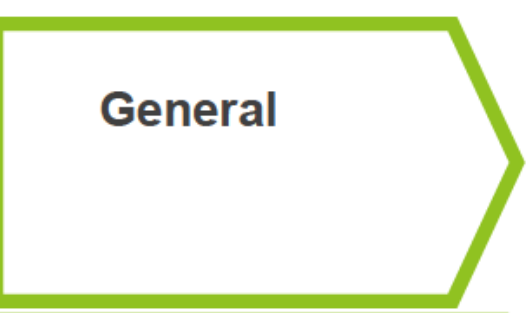

- Problem: Pre-analytical contamination (sampling, processing, material) $\Rightarrow$ Solution: negative samples
Labor methods

- Problem: Sensitivity and specificity of the culture media $\Rightarrow$ Solution: Blood cultures; Additive for species that are difficult to cultivate 
$\begin{array}{lllllllllllllllllllllll}\text { M201 } & \text { M202 } & \text { M203 } & \text { M204 } & \text { M205 } & \text { M206 } & \text { M207 } & \text { M208 } & \text { M209 } & \text { M210 } & \text { M211 } & \text { M212 } & \text { M213 } & \text { M215 } & \text { M216 } & \text { M217 } & \text { M219 } & \text { M221 } & \text { M222 } & \text { M223 } & \text { negative controls }\end{array}$

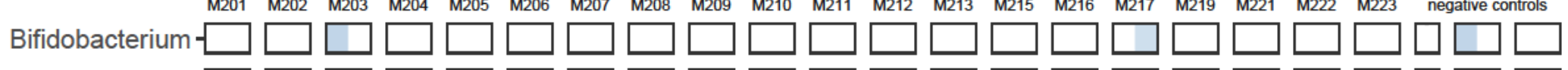
Actinobacteria Sphingomonas -
AcidocellaBradyrhizobium-

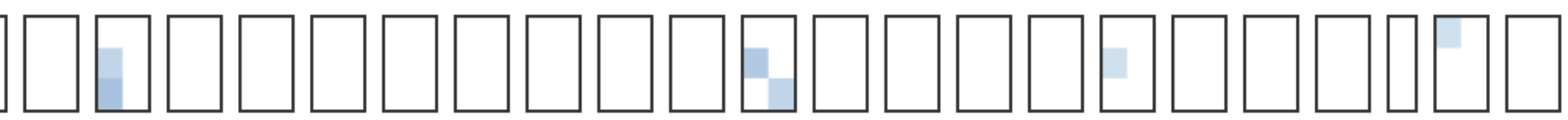

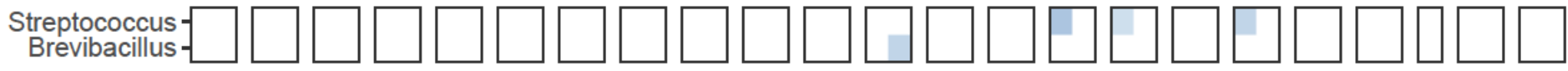
Bacteroides $\square \square \square \square \square \square \square \square \square \square \square \square \square \square \square \square \square \square \square \square \square \square \square$ Alphaproteobacteria Azorhizophilus -
Halomonas Halomonas
hodanobacter

Diaphorobacter-

Pseudomonas -

Enhydrobacter -

AcinetobacterEscherichia/ShigellaMassilia-
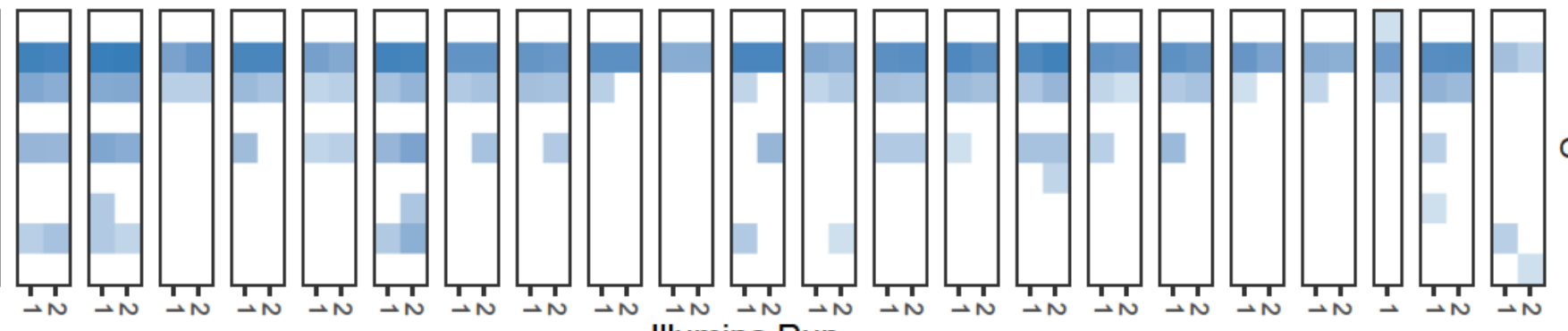

Gammaproteobacteria Illumina Run

Abundance 